Sornasse Employee of: Genentech, Inc., Michael J. Townsend Shareholder of: Stockholder of Genentech/Roche, Employee of: Genentech/ Roche, Jinglan Pei Employee of: Genentech, Margaret Michalska Employee of: Genentech, Inc.

DOI: 10.1136/annrheumdis-2019-eular.831

\section{AB0273 THE ANALYSIS OF THE CLINICAL COURSES OF THE ACPA POSITIVE PATIENTS WITHOUT SYNOVITIS: WHETHER TO FOLLOW UP THEM}

Tomoya Nakajima, Yumiko Nobuhara, Takashi Nakazawa. Osaka Saiseikai Nakatsu Hospital, Rheumatology, Osaka, Japan

Background: ACPA (anti-citrullinated peptide antibody) is a major risk factor for the onset of RA (rheumatoid arthritis) ${ }^{[1]}$. As ACPA becomes a common test in non-rheumatologists, ACPA positive patients not diagnosed with RA are increasing. However, it is unclear whether follow-up of ACPA-positive patients without synovitis leads to early treatment and consequently to the improvement of prognosis.

Objectives: To reveal whether to follow up the ACPA positive patients without synovitis.

Methods: In the four years from January 2015 to December 2018, we extracted the ACPA positive patients introduced from other hospitals, among which patients not diagnosed as RA at the first visit were selected. Then, their clinical courses and ACPA titers were retrospectively analyzed. The 2010 ACR-EULAR classification criteria was used for the diagnosis of $\mathrm{RA}^{[2]}$. For the significance test, a $\chi 2$ test and a test with $\alpha=0.05$ were used.

Results: Thirty six patients met the conditions, and then 10 patients, previously treated as RA, and 8 drop-out patients were excluded. In 18 patients remaining, 10 patients developed RA (follow-up days: avg. 404, max. 983) and the other 8 were non-RA, predisease patients (follow-up days: avg. 424, max. 926). All 10 RA patients initially started the treatment with MTX (methotrexate). Five of them reached remission with only MTX. Of the other five, three needed other csDMARDs because of an inadequate amount of MTX due to adverse events, and two had insufficient observation period. The ACPA titer was significantly higher in the RA patients than the predisease patients (468 versus $26.3 \mathrm{U} / \mathrm{ml}(P=0.0075)$ ). Tenderness of DAS 28 subject joints at the first visit was a significant predictor of RA onset when trying to extract from clinical findings. (10 of 10 in RA patients vs. 2 of 8 in predisease patients $(P=0.0044))$.

Conclusion: In our research, the predictors of RA onset are not only high ACPA titer but also tenderness of DAS28 subject joints at the first visit. The early treatment with MTX could contribute to improved prognosis, so it would be useful to follow up the ACPA positive patients without synovitis. The frequency of hospital visits and the modality to detect synovitis are the subject of our analysis from now on

\section{REFERENCES}

[1] Solbritt Rantapaa-Dahlqvist, et al.: Antibodies against cyclic citrullinated peptide and IgA rheumatoid factor predict the development of rheumatoid arthritis. Arthritis Rheum., 48 (10),2741-2749,2003.

[2] Aletaha,D.,Neogi,T.,Silman,A.,et al.: 2010 Rheumatoid arthritis classification criteria. Arthritis Rheum., 62,2569-2581,2010.

Disclosure of Interests: None declared

DOI: 10.1136/annrheumdis-2019-eular.4750

\section{AB0274 THE PREDICTIVE VALUE OF RHEUMATOID FACTOR, ANTI-CITRULLINATED PROTEIN ANTIBODIES, ANTI- CARBAMYLATED PROTEIN: ANTIBODIES AND ANTI- PEPTIDYL ARGININE DEIMINASE TYPE-3 ANTIBODIES, ALONE OR IN COMBINATION, ON RADIOGRAPHIC DAMAGE IN RHEUMATOID ARTHRITIS}

Michael Nissen $^{1}$, Céline Lamacchia ${ }^{1}$, Delphine Courvoisier ${ }^{1}$, Matthias Jarlborg ${ }^{2}$, Pascale Roux-Lombard ${ }^{2}$, Burkhard Moeller ${ }^{3}$, Adrian Ciurea ${ }^{4}$, Axel Finckh ${ }^{1}$ Chelsea Bentow ${ }^{5}$, Laura Martinez-Prat ${ }^{5}$, Michael Mahler ${ }^{5}$, Cem Gabay ${ }^{1}{ }^{1}$ Geneva University Hospital, Rheumatology, Geneva, Switzerland; ${ }^{2}$ Geneva University Hospital, Immunology, Geneva, Switzerland; ${ }^{3}$ Inselspital, Universitätsspital Bern, Bern, Switzerland; ${ }^{4}$ UniversitätsSpital Zürich, Rheumatology, Zürich, Switzerland; ${ }^{5}$ Inova Diagnostics, Inc., San Diego, United States of America

Background: Autoantibodies such as anti-citrullinated protein antibodies (ACPA), anti-carbamylated protein antibodies (CarP) and anti-peptidyl arginine deiminase 4 (PAD4) antibodies have been associated with disease severity and radiographic progression in rheumatoid arthritis $(R A)$. However, very little is known about the anti-PAD 3 (PAD3) antibodies and of the added value of combining multiple autoantibodies to predict radiographic damage.

Objectives: To investigate the capability of rheumatoid factor (RF), ACPA anti-CarP and anti-PAD3 antibodies to predict radiographic damage in RA, both individually and in combination.

Methods: We performed a nested cohort study within the "Swiss Clinica Quality Management »(SCQM) RA registry. Biobank samples were tested for RF [QUANTA Lite (QL), IgM and $\lg A$ ], ACPA IgG [QL CCP3 and QUANTA Flash (QF) CCP3], anti-CarP IgG [using carbamylated fetal calf serum as antigen by prototype ELISA, research use only (RUO)] and anti-PAD3 lgG [QF PAD3, RUO] (all methods Inova Diagnostics). Outcome: radiographic damage assessed with a validated scoring method the Ratingen (Rau) score. We examined the association of each autoantibody both separately and combined, with radiographic damage at base line and over time with linear mixed-effects models. Multivariable analyses were corrected for age, sex, smoking status, disease duration, disease activity (DAS28), number of prior biologics and calendar year of biosampling.

Figure: Radiographic damage at baseline and progression over time based on a) the presence of antiPAD3 antibodies and b) the number of autoantibodies present (RF $\operatorname{lgM}, \mathrm{QL} C \mathrm{CP} 3 \mathrm{IgG}$, anti-CarP and PAD3 antibodies and b) the number of autoantibodies present (RF $\operatorname{lgM}, \mathrm{QL} C \mathrm{CP} 3 \mathrm{IgG}$, anti-Ca
anti-PAD3). Number of patients per biomarker subgroup $-0: 177,1: 161,2: 335$ and 3-4: 178.
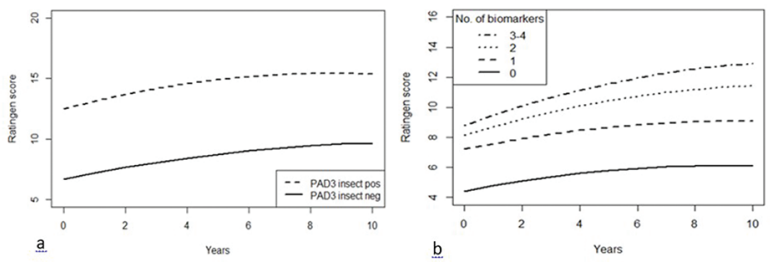

Figure 1

Results: A total of 851 RA patients were included with a median of 4 Ratingen scores per patient. Autoantibodies were positive in the following proportion of patients: RF IgM 66.3\%, RF IgA 56.9\%, QL CCP3 63.8\%, QF CCP3 63.3\%, anti-PAD3 $10.7 \%$ and anti-CarP $22.4 \%$. Significantly higher baseline Ratingen scores were associated with the presence of $\mathrm{RF}(\lg \mathrm{M}$ and $\lg \mathrm{A})$ and anti-CCP3 (QL and $\mathrm{QF})$ and greater progression over time with RF $\operatorname{lgM}$ and QL CCP3 $\lg$ ( $p=0.01$ and $p=0.04$ respectively). Patients' positive for anti-PAD3 demonstrated higher mean baseline Ratingen scores compared with anti-PAD3 negative patients (14.9 vs. 8.8 respectively) which was significant in both univariable (Figure) and multivariable analyses $(p=0.0002$ and $p=0.02$ respectively). In the $Q L$ CCP3 negative subgroup $(n=308)$, baseline Ratingen scores were significantly higher in anti-PAD3 positive patients $(p=0.01)$. There were no significant differences with regards to anti-CarP, either in the whole population or in the seronegative cohorts. The presence of multiple autoantibodies was associated with higher baseline Ratingen scores, particularly the combination of RF $\operatorname{lgM}, \mathrm{RF} \operatorname{IgA}, \mathrm{QL} C \mathrm{CP} 3$, and anti-PAD3, with a baseline Ratingen score of $16.1 \quad(p<0.00001$ compared to those with no autoantibodies). The presence of at least 3 of the following autoantibodies: RF IgM, QL CCP3, anti-CarP and anti-PAD3, was associated with significantly greater radiographic progression over 10 years (Figure) than if these autoantibodies were absent $(p=0.03)$.

Conclusion: The presence of anti-PAD3 antibodies was associated with significantly higher scores of radiographic damage at baseline, in both the overall population and in the subgroup of ACPA-negative patients. Combinations of autoantibodies (including anti-CarP and anti-PAD3) predicted both higher baseline radiographic damage and greater radiographic progression over time.

Disclosure of Interests: Michael Nissen Consultant for: AbbVie, Lilly, Novartis, and Pfizer, Céline Lamacchia: None declared, Delphine Courvoisier Grant/research support from: has received an unrestricted grant from MSD for this study, Consultant for: has received consulting fees from BMS, Pfizer, AB2 Bio and Janssen., Paid instructor for: Janssen, Matthias Jarlborg: None declared, Pascale Roux-Lombard: None declared, Burkhard Moeller Consultant for: Swissmedic Human Medicines Expert Committee Member (regulatory agency), Adrian Ciurea Consultant for: AbbVie, Celgene, Janssen-Cilag, MSD, Eli Lilly, Novartis, Pfizer, UCB, Speakers bureau: Abbvie, Celgene, Janssen-Cilag, MSD, Eli Lilly, Novartis, Pfizer, UCB, Axel Finckh Grant/research support from: Bristol-Myers Squibb, Pfizer Inc, Consultant for: AbbVie, A2Bio, Bristol-Myers Squibb MSD, Roche, Pfizer Inc, and UCB, Chelsea Bentow Employee of: INOVA Diagnostics, Laura Martinez-Prat Employee of: Inova Diagnostics (Not pharmaceutical, diagnostics company), Michael Mahler Employee of: Inova 